\title{
NTpro-BNP - diagnostic significance and recommended clinical applications
}

\author{
DUNJA ROGIĆ \\ Department of Laboratory Diagnostics, University Hospital Centre Zagreb, Zagreb, Croatia
}

Corresponding author:

Dunja Rogić

Department of Laboratory Diagnostics

University Hospital Centre Zagreb, Zagreb, Croatia

Kišpatićeva 12, 10000 Zagreb

Phone: 38512367 249; fax: 38512367395

E-mail: predstojnik.lab@kbc-zagreb.hr

\begin{abstract}
Brain natriuretic peptides are elevated in circulation in proportion to a decrease in myocardial function. They have been uniformly shown to have excellent negative predictive value in differential diagnosis of dyspnoea. They can also be used in diagnosing heart failure and have been recently shown as possible prognostic markers in acute coronary syndrome and pulmonary embolism. It is important to note that brain natriuretic peptide values rise with age and are inversely proportional to body mass index and glomerular filtration rate.
\end{abstract}

Key words: Brain natriuretic peptide $N$ terminal brain natriuretic peptide, heart failure

\section{INTRODUCTION}

Heart failure (HF) affects 1-3\% of the general population and approximately $10 \%$ of the elderly. Healthcare spending for chronic HF accounts for 1-2\% of total healthcare expenditure in developed countries. Improved survival following acute myocardial infarction (AMI) and resultant structural cardiac abnormalities have led to an increased incidence of clinical HF. Chronic HF patients have frequent short-term readmission rates, meaning that about 30\% of $\mathrm{HF}$ patients are readmitted within 30 days.

Brain natriuretic peptide was first described after being isolated from porcine brain tissue in 1988 and thus obtained its name. Soon afterwards it was established that the main biosynthesis of this peptide takes place in the cardiomyocytes within the ventricles and it steadily increases in proportion to a decrease in myocardial function. The precursor molecule is pre-proBNP which is then cleaved into proBNP, a peptide consisting of 108 aminoacids. The longer the peptide molecule, the better is its stability both in vivo and in vitro. This is why proBNP is substantially more stable in serum after blood withdrawal than BNP, which is a final biologically active molecule consisting of only 32 aminoacids. NT-proBNP is actually a biologically inactive peptide which originates as a consequence of proteolytic cleavage of proBNP by an enzyme called corin, but since it is 76 aminoacids long, it has a substantial advantage of sufficient in vitro stability as compared to BNP.

The main stimulus for increased BNP and NT-proBNP synthesis and release into the circulation is myocardial wall stress. Biochemically, its mode of action is analogous to all other peptide hormones, which is to stimulate relevant membrane receptors on target cells. BNP stimulates natriuretic peptide receptor type $\mathrm{A}$ that stimulates intracellular cyclic guanosine monophosphate production. This production in turn triggers further biochemical cascades resulting in natriuresis and diuresis, peripheral vasodilatation and inhibition of reninangiotensin and aldosterone system, which all serve to counterbalance the negative effects of deteriorating myocardial function. Being a small molecule, the in vivo half life of BNP is only 20 minutes, as compared to half life of NT-proBNP which is about 120 minutes, a fact explaining much higher normal serum values of the latter although both molecules arise in equimolar proportions. Also, the bigger size of the NT-proBNP eliminates the possibility for most of the pre-analytical mistakes, which may be a potential cause for erroneously low results due to degradation process in vitro. When converted to absolute patient numbers, the use of BNP rather than NTproBNP in an acute care setting potentially increased the false negative test results by between 0.8 and $3.1 \%$.

Since NT-proBNP is primarily excreted through kidneys, its value rises in parallel with deteriorating renal function, so this must be taken into account when evaluating the results in renal patients (figure 1)

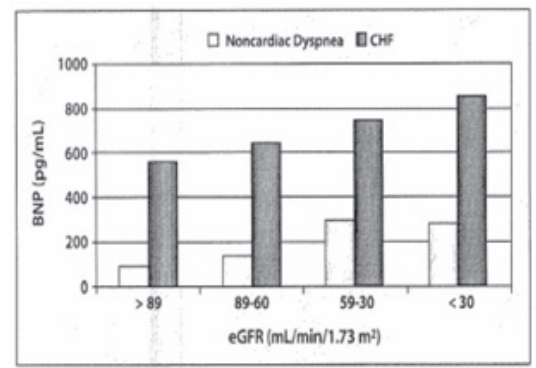

Figure 1. The relation of brain natriuretic peptide and glomerular filtration rate BNP - brain natriuretic peptide $\mathrm{CHF}$ - congestive heart failure eGFR - estimated glomerular filtration rate

Both NT-proBNP and BNP serum concentrations normally rise with age, which is why the use of age-related cut-off values has been recommended by some studies. However, even though the levels of natriuretic peptides do increase with age, this may have consequences on the test specificity, but it does not affect its sensitivity, i.e. its excellent ability to serve as a heart failure rule-out test.

Both BNP and NT-proBNP have high week-to-week intra-individual coefficients of variation of $113 \%$ and $98 \%$, respectively, with the biological variation being consist- 
ently higher for BNP probably due to its shorter half life in circulation.

It is also very important to take into account the patient's BMI because the values in obese patients have consistently been shown to be significantly lower than in normal weight patients. In other words, they are inversely proportional to BMI (figure 2), so there is a danger of misclassifying obese patients on the basis of their natriuretic peptide value.

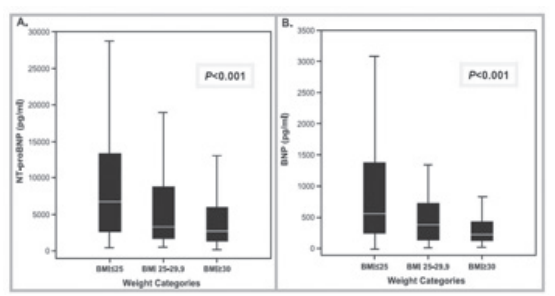

Figure 2. NT-proBNP and BNP value distribution according to BMI

BMI - body mass index

BNP - brain natriuretic peptide

NT-proBNP - N-terminal pro brain natriuretic peptide

\section{DIAGNOSTIC SIGNIFICANCE}

In the following clinical situations, the above mentioned effects of deteriorated renal function, obesity and/or advancing age must be taken into account.

\section{Acute dyspnoea - differential diag- nosis}

The most common causes of unclear dyspnoea in the emergency department are congestive heart failure (CHD), chronic obstructive pulmonary disease (COPD), acute coronary syndrome (ACS), asthma, pneumonia and pulmonary embolism. It has been clearly established that natriuretic peptides demonstrate excellent sensitivities, i.e. negative predictive values in excluding myocardial causes of dyspnoea. The newest systematic reviews point out that negative likelihood ratios for both BNP and NT-proBNP are around 0.12. Of course, if the clinical scenario is fairly clear and strongly suggestive of heart failure or, conversely, points to an alternative diagnosis, there is no need to perform the assay. It also has to be taken into account that newer studies point to the fact that natriuretic peptides are often markedly elevated in cases of pulmonary embolism, so a positive value is not heart-specific.

\section{Diagnosing heart failure}

The diagnosis of heart failure (HF) is often challenging, as the symptoms and signs may be non-specific and may occur in other non-cardiac diseases. The mortality from HF remains high, at approximately $25 \%$ over one year, which could be reduced through prompt diagnosis and treatment. The 2012 European Society of Cardiology guidelines for heart failure include specific age-independent decision limits for natriuretic peptides for the exclusion of acute heart failure (NT-proBNP $\leq 300$ ng/L, BNP $\leq 100$ ng/L). Conversely, HF inclusion criteria for NT-proBNP are agedependent, as shown in table 1.

Although natriuretic peptide levels have been used widely for the early diagnosis or exclusion of chronic heart failure in the outpatient setting, their use in the acute care setting has only partially been adopted. However, these tests have excellent ability to exclude the diagnosis of acute heart failure, with sensitivities approaching 1 and will therefore hardly misclassify any cases of possible acute heart failure when used as a rule-out test. A negative test result will reduce or eliminate the need to proceed to echocardiography or other imaging methods, allowing a more efficient use of resources. On the other hand, the specificity is relatively low (about 60\%, although it increases with increasing numerical value), thus confirmatory testing by cardiac imaging is required. It is therefore of utter importance for natriuretic peptide values which fall above the rule- out thresholds to correlate the information with other diagnostic and clinical data in order to exclude non-cardiac causes of elevated results, such as pulmonary embolism, sepsis or renal failure.

\section{Emerging data: risk stratification - acute coronary syndrome and pulmo- nary embolism}

The spectrum of acute coronary syndrome (ACS), from ST segment elevation myocardial infarction to unstable angina without irreversible myocardial necrosis, induces increased plasma concentrations of natriuretic peptides. Activation of the BNP gene is caused by acute ischemia with tissue hypoxia as well as by increased myocardial stretch secondary to ischemiainduced left ventricular systolic and/or diastolic dysfunction. Multiple biomarkers at admission significantly improve the prediction of mortality in patients undergoing primary percutaneous coronary intervention for acute ST-segment elevation myocardial infarction and it has been shown that NT-proBNP above $600 \mathrm{ng} / \mathrm{L}$ doubles the mortality risk.

Several studies performed in patients with acute pulmonary embolism have demonstrated that NT-proBNP value was associated with the likelihood for death or escalation of therapy with an odds ratio of nearly 15 , and a negative predictive value of $97 \%$. Essentially, the lower the values of NT-proBNP are, the lower is the likelihood for complications.

\section{CONCLUSION}

Both BNP and NT-proBNP have been shown to represent added value in differential diagnosis of dyspnoea, as well as in diagnosing and monitoring heart failure. Recent studies also point at their possible prognostic significance in acute coronary syndrome and pulmonary embolism. 
Table 1, NT-proBNP values in heart failure

\begin{tabular}{lll}
\hline Heart failure unlikely & Grey zone & Heart failure likely \\
\hline$\leq 300$ & - send patient to imaging & - confirm by imaging \\
\hline & In between 300 and age-related & $>450$ below 50 years of age \\
& inclusion cut-off & $>900$ between $50-75$ \\
& $>1800$ above 75 \\
\hline
\end{tabular}

\section{REFERENCES}

1. Roberts E, Ludman AJ, Dworzynski K, Al-Mohammad A, Cowie MR, McMurray JJV, Mant J (on behalf of the NICE Guideline Development Group for Acute Heart Failure). The diagnostic accuracy of the natriuretic peptides in heart failure: systematic review and diagnostic meta-analysis in the acute care setting. BMJ 205;350:h910 doi: 10.1136/bmj.h910.

2. Yancy CW, Jessup M, Bozkurt B, Butler J, Casey DE, Drazner M, et al. ACCF/AHA guideline for the management of heart failure: a report of the American College of Cardiology Foundation/American Heart Association Task Force on practice guidelines. Circulation 2013;128:e240-327.

3. McMurray JJV, Adamopoulos S, Anker SD, Auricchio A, Bohm M, Dickstein K, et al. ESC guidelines for the diagnosis and treatment of acute and chronic heart failure 2012: The Task Force for the Diagnosis and Treatment of Acute and Chronic Heart Failure 2012 of the European Society of Cardiology. Developed in collaboration with the Heart Failure Association (HFA) of the ESC. Eur J Heart Fail 2012;14:803-69.

4. Thygesen K1, Mair J, Mueller C, Huber K, Weber M, Plebani M, et al. Recommendations for the use of natriuretic peptides in acute cardiac care: a position statement from the Study Group on Biomarkers in Cardiology of the ESC Working Group on Acute Cardiac. Care Eur Heart J 2012;33;2001-6.

5. Novielli, N, Cooper NJ, Abrams KR, Sutton AJ. How is evidence on test performance synthetized for economic decision models of diagnostic tests? A systematic appraisal of Health Technology Assessments in the UK since 1997. Value Health 2010;13:952-7.

6. Chenevier-Gobeaux C, Guerin S, Andre S, Ray P, Cynober L, Gestin S, et al. Midregional pro-atrial natriuretic peptide for the diagnosis of cardiac-related dyspnea according to renal function in the emergency department: a comparison with B-type natriuretic peptide (BNP) and N-terminal proBNP. Clin Chem 2010;56:1708-17.

7. Nazerian P, Vanni S, Zanobetti M, Polidori G, Pepe G, Federico R, et al. Diagnostic accuracy of emergency Doppler echocardiography for identification of acute left ventricular heart failure in patients with acute dyspnea: comparison with Boston criteria and N-terminal prohormone brain natriuretic peptide. Acad Emerg Med 2010;17:18-26. 